doi: https://doi.org/10.15407/dopovidi2018.08.045

UDC 532.595

\author{
A.N. Timokha, Corresponding Member of the NAS of Ukraine \\ Institute of Mathematics of the NAS of Ukraine, Kiev \\ Centre of Excellence "Autonomous Marine Operations and Systems", \\ Department of Marine Technology, Norwegian University \\ of Science and Technology, Trondheim, Norway \\ E-mail: tim@imath.kiev.ua, atimokha@gmail.com

\section{Prandtl steady rotary current in an upright cylindrical container}

The quantifying of the experimentally-known (Prandtl, 1949) steady rotary current during the swirl-type sloshing is first given. The current is treated as the sum of the mean wave (pseudo-) momentum through the meridional crosssection and the mean wave Eulerian flow, which is governed by the Craik-Leibovich equation. The constructed analytical inviscid theory is supported by existing experimental data.

Keywords: sloshing, swirling wave, Craik-Leibovich equation, Stokes drift.

In 1949, Ludwig Prandtl conducted a dedicated model test on the resonant sloshing in an orbitally-shaken upright circular tank to show that the swirl-type sloshing yields a steady circular rotation of the contained liquid [1]. The phenomenon was later reproduced by Hutton [2] and, recently, in [3-5], mainly, in context of sloshing in bioreactors. The steady rotary current occurs, for each inner liquid point, strictly in the angularly-propagating wave direction; its (rotary current) velocity is zero on the wall and reaches its maximum about the semiradius. To date with, the three aforementioned peculiarities of the rotary current are not theoretically quantified. Existing theoretical studies associate the Prandtl phenomenon with the angular Stokes drift and, in parallel way, discuss the steady streaming effect. Proceeding this way may, generally, clarify an angular fluid mass-transport in the swirling wave direction, but other two peculiarities of the steady rotary current remain unexplained.

Employing the asymptotic steady-state wave resonant-sloshing solution from [6], in general, and adopting its lowest-order inviscid irrotational-flow component as an ambient flow, in particular, the present paper proposes an inviscid analytical theory of the steady rotary (Prandtl) current, which is interpreted, over here, as the direct sum of the mean wave (pseudo-) momentum per unit area of the meridional cross-section and the mean wave Eulerian (vortical) flow. The latter is governed by the Craik-Leibovich equation and implies the counter-directed (relative to the pseudo-momentum) fluid mass-transport.

(C) A.N. Timokha, 2018 


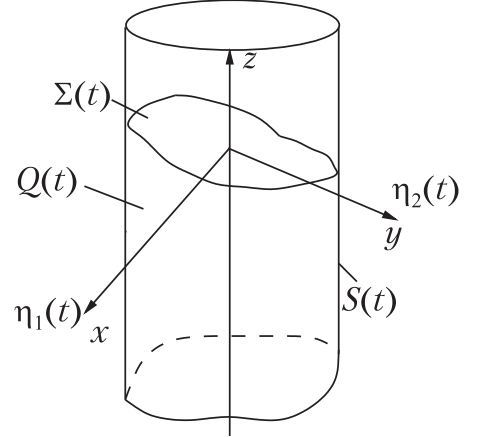

$a$

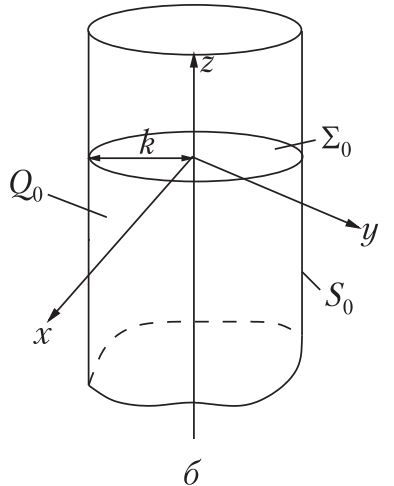

Fig. 1. The upright circular container with an infinite liquid depth moves translatorily along a horizontal elliptic orbit defined by $\eta_{1}(t)=$ $=\eta_{1 a} \cos \sigma t$ and $\eta_{2}(t)=\eta_{2 a}$ sin $\sigma t$. Sloshing is considered in the tank-fixed coordinate system so that $\mathrm{Oz}$ coincides with the symmetry axis, and $z=0$ determines the mean free surface. The panel $(a)$ introduces geometric notations and ( $b$ ) shows the hydrostatic (mean) liquid shape as it appears for the adopted normalisation

An upright circular cylindrical rigid tank of radius $r_{0}$ performs a small-magnitude prescribed periodic (elliptic) horizontal motion, which is described by the two generalised coordinates $\eta_{1}(t)=\eta_{1 a} \cos \sigma t$ and $\eta_{2}(t)=\eta_{2 a} \sin \sigma t$ with the forcing frequency $\sigma$ close to the lowest natural sloshing frequency $\sigma_{1}=\sigma_{11}$ (fig. 1). For the infinite liquid depth $\left(h / r_{0} \rightarrow \infty\right)$, the natural frequencies are computed by $\sigma_{M i}^{2}=g k_{M i} / r_{0}$, where $g$ is the gravity acceleration, and $k_{M i}$ are the roots of $J_{M}^{\prime}\left(k_{M i}\right)=0\left(J_{M}\right.$ is the Bessel function of the first kind).

When following [6], one can construct the Narimanov-Moiseev-type steady-state wave asymptotic solution of the resonant sloshing problem by assuming a small-amplitude tank excitation, $\left(\eta_{1 a}^{2}+\eta_{2 a}^{2}\right) / r_{0}^{2}=O\left(\varepsilon^{2}\right) \ll 1$, and requiring the so-called Moiseev detuning $\left|\sigma^{2}-\sigma_{1}^{2}\right| / \sigma^{2}=$ $=O\left(\varepsilon^{2 / 3}\right)$. For brevity, we adopt the normalisation, in which $1 / \sigma$ and $r_{0} / k$ are the characteristic time and size, respectively $\left(k=k_{11}=1.841183781341 \ldots\right)$. According to [6], the lowestorder asymptotic approximation of the steady-state wave free-surface $\Sigma(t)$ representation, $z=\zeta(r, \theta, t)=\zeta^{(1 / 3)}+\zeta^{(2 / 3)}+\ldots$, takes the form

$$
\zeta^{(1 / 3)}(r, \theta, t)=J_{1}(r)\left(-\theta_{s} \cos t+\theta_{c} \sin t\right)
$$

where $\theta_{c}=b \sin \theta+\bar{a} \cos \theta, \theta_{s}=-\bar{b} \cos t-a \sin t$, and $a, \bar{a}, \bar{b}, b=O\left(\varepsilon^{1 / 3}\right)$ are the four non-dimensional amplitude parameters, which come from a nonlinear secular system [6]. Depending on the quadratic quantity $\Xi=a b-\bar{a} \bar{b}$, formula (1) determines either a standing (only for longitudinal excitations) or swirling (angular progressive) wave, i.e., $\Xi=0 \Leftrightarrow$ standing, $\Xi>0 \Leftrightarrow$ counterclockwise swirling, and $\Xi<0 \Leftrightarrow$ clockwise swirling. The asymptotic solution also represents the relative velocity field $\boldsymbol{v}=v_{1} \hat{\boldsymbol{r}}+v_{2} \hat{\boldsymbol{\theta}}+v_{3} \hat{\boldsymbol{z}}=\boldsymbol{v}^{(1 / 3)}+\boldsymbol{v}^{(2 / 3)}+\ldots$, where $\hat{\boldsymbol{r}}, \hat{\theta}, \hat{\boldsymbol{z}}$ are the cylindrical coordinate frame units and

$$
\boldsymbol{v}^{(1 / 3)}(r, \theta, z, t)=\nabla\left[J_{1}(r) e^{z}\left(\theta_{c} \cos t+\theta_{s} \sin t\right)\right]=v_{1}^{(1 / 3)} \hat{\boldsymbol{r}}+v_{2}^{(1 / 3)} \hat{\theta}+v_{3}^{(1 / 3)} \hat{\boldsymbol{z}} .
$$

The inviscid potential flow implies the zero mean (time-averaged) velocity field, i.e., $\langle\boldsymbol{v}\rangle=0$. On the other hand, according to [7, Sect. 9.6.3], the mean angular wave (pseudo-) momentum per unit area of the meridional cross-section (determined by the potential-flow solution),

$$
M=\left\langle\int_{-\infty}^{\zeta} \int_{0}^{k} v_{2} \hat{\theta} d r d z\right\rangle=\int_{0}^{k}\left\langle\left. v_{2}^{(1 / 3)}\right|_{z=0} \zeta^{(1 / 3)}\right\rangle d r \hat{\theta}+o\left(\varepsilon^{2 / 3}\right)=\Xi \int_{0}^{k} \frac{J_{1}^{2}(r)}{2 r} d r \hat{\theta}+o\left(\varepsilon^{2 / 3}\right),
$$


is linearly proportional to $\Xi$ and, therefore, is non-zero for the swirl-type sloshing. The latter fact means that the contained liquid slowly rotates, even though we adopt the inviscid potential-flow hydrodynamic model. The meridional distribution of the mean wave momentum can be derived as

$$
\boldsymbol{w}^{M}(r, z)=\Xi e^{2 z} w^{M}(r) \hat{\theta}=\Xi e^{2 z}\left(r^{-1} J_{1}^{2}(r)\right) \hat{\theta} .
$$

Specifically, $\boldsymbol{w}^{M}$ does not coincide with the second-order Stokes drift velocity

$$
\begin{aligned}
& \boldsymbol{w}^{S}(r, z)=\left\langle\int \boldsymbol{v}^{(1 / 3)} d t \cdot \nabla \boldsymbol{v}^{(1 / 3)}\right\rangle=\Xi e^{2 z} w^{S}(r) \hat{\boldsymbol{\theta}}= \\
& =\Xi e^{2 z}(2 r)^{-1}\left[J_{1}^{2}(r)+\left(J_{1}^{\prime 2}(r)-r^{-1} J_{1}(r)\right)^{2}\right] \hat{\boldsymbol{\theta}},
\end{aligned}
$$

as it happens for the two-dimensional propagating wave.

Neither $w^{M}$ nor $w^{S}$ can quantify the aforementioned peculiarities of the steady rotary current. Using $w^{M}$ and/or $w^{S}$ only theoretically states that a fluid mass-transport in the swirling wave direction is possible within the framework of Eulerian and/or Lagrangian specification, respectively.

Henceforth, we assume that there exists a non-zero mean wave angular vortex (Eulerian) flow $\boldsymbol{w}^{E}=\langle\boldsymbol{v}\rangle, \omega^{E}=\nabla \times \boldsymbol{w}^{E} \neq 0$, which was not accounted in [6]. By an analogy to [8], the steady vortex flow appears as a second-order, $O\left(\varepsilon^{2 / 3}\right)$, supplement to $\boldsymbol{v}$ by [6]. Utilising the vorticity equation in the tank-fixed coordinate system and the time-averaging technique leads, after long and very tedious derivations, to the Craik-Leibovich equation and the continuity equations

$$
\nabla \times\left[\left(\boldsymbol{w}^{E}+\boldsymbol{w}^{S}\right) \times \omega^{E}\right]=0 \text { and } \nabla \cdot \boldsymbol{w}^{E}=0
$$

in the mean liquid domain $Q_{0}$, which should be equipped with the boundary conditions

$$
\left.w_{1}^{E}\right|_{r=k}=0 \quad \text { and }\left.\quad w_{3}^{E}\right|_{z=0}=0 \text {. }
$$

The homogeneous boundary-value problem, has two trivial solutions. The first solution $\boldsymbol{\omega}^{E}=\mathbf{0} \Rightarrow \boldsymbol{w}^{E}=\mathbf{0}$ implies the zero mean vortex flow. This is not consistent with experiments [2-5] and our theoretical expectations. The second solution states that the mean Lagrangian flow is zero, $\boldsymbol{w}^{L}=\boldsymbol{w}^{S}+\boldsymbol{w}^{E}=\mathbf{0}$. This solution is derivable (see Remark 1) from and when postulating $\boldsymbol{w}^{L}=\mathbf{0} \Rightarrow \boldsymbol{w}^{E}=-\boldsymbol{w}^{S}$ at $r=k$. In other words, when assuming that the Prandtl phenomenon is associated with the mean Lagrangian fluid motions, $\boldsymbol{w}^{L}$, the zero Lagrangian velocity on the wall implies $\boldsymbol{w}^{L}=\mathbf{0}$ in the whole domain, i.e., we arrive at the contradiction - the steady rotary current does not exist within the framework of the inviscid hydrodynamic model. Our forthcoming focus is therefore on the sum $\boldsymbol{w}^{P}=\boldsymbol{w}^{M}+\boldsymbol{w}^{E}$.

To obtain a unique non-trivial solution of (6), (7), one should postulate an extra tangential boundary condition for $\boldsymbol{w}^{E}$ on the wall. Experiments [2,5] say us that the rotary current velocity tends to zero at the wall, namely, they require the non-slip boundary condition $\boldsymbol{w}^{P}=\mathbf{0} \Rightarrow$ $\Rightarrow \boldsymbol{w}^{E}=-\boldsymbol{w}^{M}$ on $S_{0}$. 
The no-slip condition is an attribute of viscous flows. A paradox is that this condition is natural in our inviscid analysis and, as we will show, leads to a satisfactory description of the mean flow phenomenon. Why is it so? Adopting $\boldsymbol{w}^{E}=-\boldsymbol{w}^{M}$ on $S_{0}$ implies that the steady rotary current by $\boldsymbol{w}^{P}=\boldsymbol{w}^{M}+\boldsymbol{w}^{E}$, if exists, is not affected, itself, by frictional forces on the wall, simply, because fluid particles do not steadily move along the tank surface. What is the physical origin of $\boldsymbol{w}^{E}$ ? Why does it counteract $\boldsymbol{w}^{M}$ ? Most probably, $\boldsymbol{w}^{E}$ is initiated by the so-called steady streaming, which can be described within the framework of the nonlinear boundary-layer theory [9]. According to the steady-streaming prediction [9], $w^{E}$ should be counter-directed to $w^{M}$ at the wall, namely, it determines a return steady flow. However, the steady streaming theory [9] does not cause $\boldsymbol{w}^{P}=\mathbf{0}$ at the wall. An extra frictional steady force on the wall is then expected, which should increase the return flow by $\boldsymbol{w}^{E}$ until the no-slip condition for $\boldsymbol{w}^{P}$ would be fulfilled. When this finally happens, $\boldsymbol{w}^{P}=\boldsymbol{w}^{M}+\boldsymbol{w}^{E}$ becomes unaffected by frictional forces at the wall and, therefore, the Prandtl phenomenon could be modelled within the framework of the inviscid hydrodynamic model.

Postulating $\boldsymbol{w}^{P}=\mathbf{0} \Rightarrow \boldsymbol{w}^{E}=-\boldsymbol{w}^{M}$ on the wall causes an axisymmetric solution of the boundary-value problem (6), (7). Requiring a continuous finite vector-function $w^{E}$ in $Q_{0}$, the $\hat{\boldsymbol{r}}$ and $\hat{\boldsymbol{z}}$ scalar components of the Craik-Leibovich equation in (6) deduce that $\partial_{z} G=0$ \& $\left(r \partial_{r} G+G\right)=0$, where $G=w_{1}^{E} \omega_{3}^{E}-w_{3}^{E} \omega_{1}^{E}$, and $\Rightarrow G=C / r \Rightarrow C=0 \Rightarrow G=0 \Rightarrow$

$$
w_{1}^{E} \omega_{3}^{E}-w_{3}^{E} \omega_{1}^{E}=\partial_{z}\left(r w_{2}^{E}\right) w_{3}^{E}+\partial_{r}\left(r w_{2}^{E}\right) w_{1}^{E}=0,
$$

but the continuity equation takes the form

$$
\partial_{z}\left(r \omega_{3}^{E}\right)+\partial_{r}\left(r \omega_{1}^{E}\right)=0
$$

Because we suggest a non-trivial angular flow component $w_{2}^{E}$, solving (7), (8), (9) with respect to the pair $w_{1}^{E}, w_{3}^{E}$ leads to the zero solution (just applying the method of characteristics to (9) with constraint (8)). The remaining $\hat{\theta}$-component of the Craik-Leibovich equation in (9) together with the no-slip condition $\boldsymbol{w}^{E}=-\boldsymbol{w}^{M}$ on the wall constitute the boundaryvalue problem

$$
\begin{aligned}
& 2 \omega_{2}^{E} \partial_{z} \omega_{2}^{z}+\Xi e^{2 z}\left[3\left(2 w^{S}-w^{M}\right) \partial_{z} w_{2}^{E}+2 w^{S} \partial_{r}\left(r \omega_{2}^{E}\right)\right]=0 ;\left.w_{2}^{E}\right|_{r=k}=-\Xi e^{2 z} p_{0} ; \\
& \left.w_{2}^{E}\right|_{r=k}=-\Xi e^{2 z} p_{0} ;\left|\omega_{2}^{E}\right|_{r=0} \mid<\infty
\end{aligned}
$$

where $p_{0}=J_{1}^{2}(k) / k$. The exact analytical solution of (10) takes the form

$$
w_{2}^{E}=\Xi e^{2 z} w^{E}(r), \quad w^{E}(r)=\frac{\omega^{S}(r)}{c_{0} r^{2}-1}, \quad c_{0}=\frac{1}{k^{2}}\left(1-\frac{w^{S}(k)}{p_{0}}\right) .
$$

Remark 1. If $p_{0}=w^{S}(k) \Rightarrow c_{0}=0 \Rightarrow w^{E}(r)=-\omega^{S}(r)$, and, therefore, we arrive at the trivial solution $w^{L}(r)=0 \Rightarrow \boldsymbol{w}^{L}=\mathbf{0}$, which was discussed in context of the boundary-value problem (6), (7). 

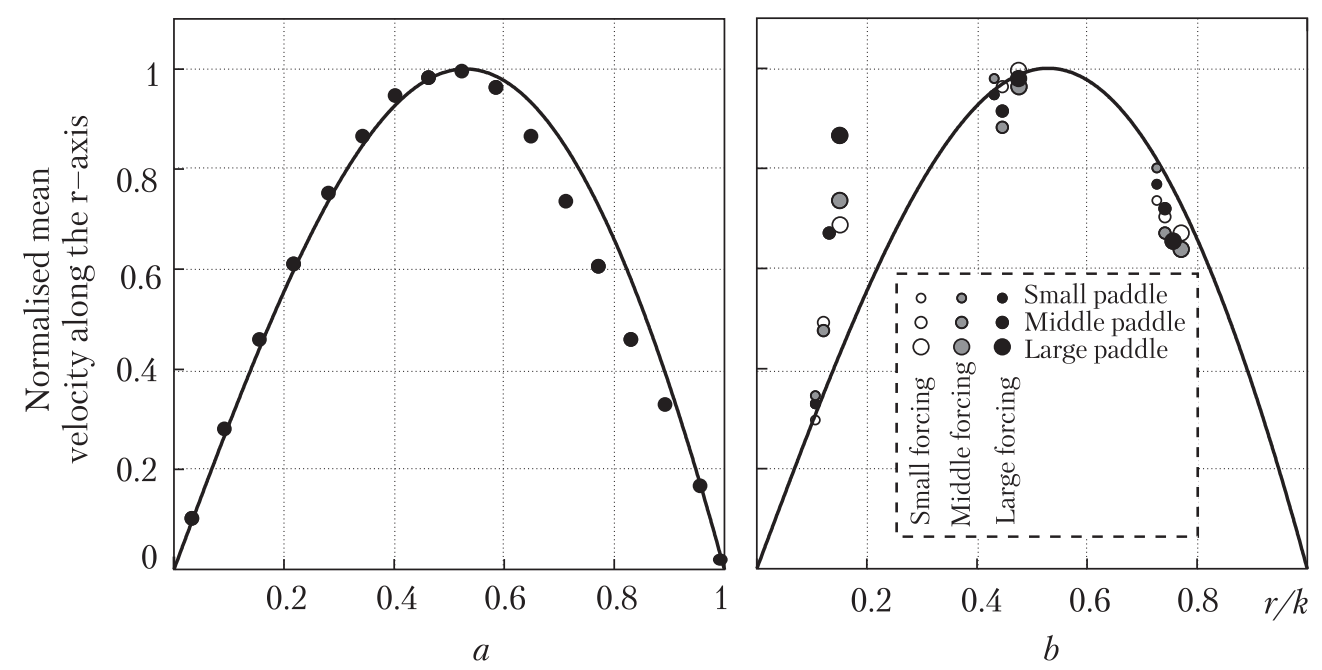

Fig. 2. The measured ([2,5], circles) and theoretical (by (12), the solid lines) mean wave velocity $w^{P}(r) / \max _{r}\left|w^{P}(r)\right|$. Panel (a) implies measurements [5] made for the orbital forcing, $\sigma / \sigma_{1}=0.78, z_{0}=-0.1061 h\left(h / r_{0}=2.168\right.$, $\left.r_{0}=51.2 \mathrm{~mm}\right), R_{s}=r_{0}^{2} \sigma / \mathrm{v}=1000$. Panel $(b)-$ experiments [2] with the longitudinal forcing, $R_{s}=4.5 \cdot 10^{5}$, $h / r_{0}=1.4, r_{0}=22.47 \mathrm{~cm}, \sigma / \sigma_{1}=1, z_{0}=-0.422 h$; three different forcing amplitudes ("small", "middle", "large") were used, which are not specified in [2] but associated with experimental crest/trough at the wall equal to 8.89/5.588, 11.43/6.35, 13.97/7.62 cm, respectively; mechanical measurements adopted rectangular paddles of different sizes ("small paddle" $=l_{r} / l_{z}=3.937 \times 2.286 \mathrm{~cm}$, "medium paddle" $=4.8514 \times 3.6068 \mathrm{~cm}$, "large padd$\mathrm{le}=5.968 \times 4.334 \mathrm{~cm})$.

The mean rotary current is, according to our inviscid theory, described by

$$
\boldsymbol{w}^{P}(r, z)=\Xi e^{2 z} w^{P}(r) \hat{\theta}=\Xi e^{2 z}\left(\frac{J_{1}^{2}(r)}{r}+\frac{w^{S}(r)}{c_{0} r^{2}-1}\right) \hat{\theta},
$$

where $\omega^{S}(r)$ is defined by (5) and $c_{0}$ is computed in (11). The theoretical prediction of $\omega^{P}(r) / \max _{r}\left|w^{P}(r)\right|$ by (12) can then be compared with the corresponding experimental data from $[2,5]$ made at the fixed vertical level $z=z_{0}$ beneath the mean free surface. The result is presented in Fig. 2.

Using the PIV velocimetry, [5] measured the fluid mass-transport velocity through the meridional plane for the swirl-type sloshing in a circular container, which is exposed to the circular orbital forcing. The container was filled by silicon oil with the Reynolds number $R_{s}=r_{0}^{2} \sigma / v=1000$ ( $v$ is the kinematic viscosity). The liquid depth was $h / r_{0}=2.168\left(r_{0}=51.2 \mathrm{~mm}\right)$ and several forcing frequencies were applied, one of which, $\sigma / \sigma_{1}=0.78$, was sufficiently close to the primary resonance to apply the asymptotic resonant sloshing theory from [6]. The measurements were done at the vertical level $z_{0}=-0.1061 \mathrm{~h}$. The experimental mean wave velocities were normalised by its maximum value, which was found approximately at $\frac{1}{2} r_{0}$. The experimental and theoretical functions $w^{P}(r) / \max \left|\omega^{P}\right|$ are compared in Fig. 2. A satisfactory agreement is found.

Another experimental series was reported in [2] for the longitudinal excitation with the forcing frequency equal to $\sigma_{1}=8.91 \mathrm{rad} \mathrm{s}^{-1}$, when the swirling wave mode is stable [6]. This case is 
analysed in Fig. $2(b)$. Three different experimental forcing amplitudes were utilised, which were not specified in [2] but, instead, the output data were distinguished by documenting the corresponding experimental crest and through at the wall. The geometric input is $r_{0}=22.47 \mathrm{~cm}$ and $h=31.45 \mathrm{~cm}$. To measure the mean wave velocity distribution, Hutton in [2] used a mechanical device, whose main element is a rectangular paddle lying in the meridional plane and rotating together with the current. Three different paddles were employed. The measurements were made at the vertical level $z_{0}=-0.422 h$, which is fairly deep and far away from the mean free surface. The experimental values are marked by circles. The smaller symbol means the smaller paddle size. The grey filling depth of the circles increases with increasing the forcing amplitude. Fig. $2(b)$ supports our analytical inviscid theory for measurements with the "small paddle". The "medium" and "large" paddles give larger velocity values for the measurement probe, which is close to the tank centre.

In summary, the Prandtl phenomenon consisting of a mean angular fluid rotation during the swirl-type resonant sloshing in a vertical circular cylindrical tank with an infinite liquid depth is first theoretically quantified by utilising the asymptotic steady-state wave solution from [6]. In the constructed inviscid theory, the steady rotary current is associated with the summarised effect of the mean wave (pseudo-) momentum through the meridional plane and the mean vortical (Eulerian) flow. To find the last component, the Craik-Leibovich equation for the swirling wave mode is derived and analytically solved. The solution requires the tangential boundary condition on the vertical wall. Adopting the no-slip condition for the summarised mean fluid rotation, which becomes natural for our inviscid theory, leads to an exact analytical expression, which determines the summarised steady current velocity. The latter result is in a satisfactory agreement with measurements $[2,5]$.

The author acknowledges the financial support of the Centre of Autonomous Marine Operations and Systems (AMOS), whose main sponsor is the Norwegian Research Council (Project number 223254-AMOS).

\section{REFERENCES}

1. Prandtl, L. (1949). Erzuengung von Zirkulation beim Schütteln von Gefässen. ZAMM, 29, No. 1/2, pp. 8-9.

2. Hutton, R. E. (1964). Fluid-particle motion during rotary sloshing. J. Appl. Mech., Transactions ASME, 31, No. 1, p. 145-153.

3. Royon-Lebeaud, A., Hopfinger, E.J. \& Cartellier, A. (2007). Liquid sloshing and wave breaking in circular and square-base cylindrical containers. J. Fluid Mech., 577, pp. 467-494.

4. Reclari, M. (2013). Hydrodynamics of orbital shaken bioreactors. Ecole Polytechnique Federale de Lausanne, PhD Thesis, No. 5759, 159 pp.

5. Bouvard, J., Herreman, W. \& Moisy, F. (2017). Mean mass transport in an orbitally shaken cylindrical container. Phys. Review, Fluids, 2, No. 084801, pp. 1-17.

6. Faltisen, O. M., Lukovsky, I. A. \& Timokha, A. N. (2016). Resonant sloshing in an upright annular tank. J. Fluid Mech. 804, pp. 608-645.

7. Faltisen, O. M., Lukovsky, I. A. \& Timokha, A. N. (2009). Sloshing. Cambridge Univ. Press.

8. Craik, A. D. \& Leibovich, S. (1976). A rational model for Langmuir circulations. J. Fluid Mech., 73, No. 3, pp. 401-426.

9. Timokha, A. N. (2018). Nonlinear boundary-layer and laminar vertical stream generated by resonant sloshing in a circular-base tank. Nonlinear Oscillations, 21, No. 1, pp. 131-145.

Received 06.05.2018 


\section{O.M. Tимоха}

Інститут математики НАН України, Київ

Центр досконалості “Автономні морські операції та системи”,

Департамент морських технологій, Норвезький університет природничих

та технічних наук, Трондхейм, Норвегія

E-mail: tim@imath.kiev.ua, atimokha@gmail.com

\section{КРУГОВИЙ УСТАЛЕНИЙ ПОТІК ПРАНДТЛЯ}

\section{У ВЕРТИКАЛЬНОМУ ЦИЛІНДРИЧНОМУ КОНТЕЙНЕРІ}

Вперше дається кількісне визначення експериментально відомого усталеного кругового потоку Прандля (1949), який виникає під час кругової хвилі в контейнері. Потік пов'язується із сумарним ефектом (псевдо-) імпульса крізь меридіональну площину та усередненою хвильовою ейлерівською течією, яка описується рівнянням Крейка-Лейбовича. Побудована аналітична нев'язка теорія підтверджується існуючими експериментальними даними.

Ключові слова: хлюпання рідини, кругова хвиля, рівняння Крейка-Лейбовича, дрейф Стокса.

\section{A.H. Тимоха}

Институт математики НАН Украины, Киев Центр совершенства “Автономные морские операции и системы”, Департамент морских технологий, Норвежский университет естественных и технических наук, Трондхейм, Норвегия E-mail: tim@imath.kiev.ua, atimokha@gmail.com

\section{КРУГОВОЙ УСТАНОВИВШИЙСЯ ПОТОК ПРАНДЛЯ В ВЕРТИКАЛЬНОМ ЦИЛИНДРИЧЕСКОМ КОНТЕЙНЕРЕ}

Впервые дается количественное определение экспериментально известного установившегося кругового потока Прандтля (1949), который возникает во время круговой волны в контейнере. Поток связывается с суммарным эффектом (псевдо-) импульса через меридиональное сечение и усредненным волновым эйлеровским течением, которое описывается уравнением Крейка-Лейбовича. Построенная аналитическая теория подтверждается существующими экспериментальными данными.

Ключевые слова: плескание жидкости, уравнение Крейка-Лейбовича, дрейф Стокса. 